\title{
Artificial Neural Network Algorithm for On-Line Glucose Prediction from Continuous Glucose Monitoring
}

\author{
C. Pérez-Gandia ${ }^{1,2}$, A. Facchinetti ${ }^{3}$, G. Sparacino ${ }^{3}$, \\ C. Cobelli ${ }^{3}$, E.J. Gómez ${ }^{2,1}$, M. Rigla ${ }^{4}$ A. de Leiva ${ }^{5}$, M.E. Hernando ${ }^{2,1}$ \\ 'Networking Research Center on Bioengineering, Biomaterials and Nanomedicine \\ (CIBER-BBN), Madrid, Spain. \\ ${ }^{2}$ Grupo de Bioingeniería y Telemedicina, Universidad Politécnica de Madrid, Spain. \\ ${ }^{3}$ Dipartimento di Ingegneria dell'Informazione, Università degli Studi di Padova, Italy. \\ ${ }^{4}$ Endocrinology Department, Hospital de Sabadell, Spain. \\ ${ }^{5}$ Endocrinology Department, Hospital Sant Pau, Barcelona, Spain.
}

\section{Funding source:}

This work was partially supported by the Spanish FIS grant from the Ministry of Health and Consumer Affairs "ADVISING"- FIS PI060437 and CIBER-BBN (PREDIRCAM) and by the grant "Simulation models and filtering/prediction techniques for the development of an artificial pancreas" from the Italian Ministry of Education. University and Research (PRIN 2007).

\section{Corresponding Author: Carmen Pérez-Gandía}

$\begin{array}{ll}\text { Address: } & \text { Gnupo de Bioingeniería y Telemedicina, ETSI Telecomunicación. Ciudad Universitaria } \\ & \text { s/n, 28040 MADRID, Spain } \\ \text { Phone } & +34915495700 \\ \text { Fax } & +34913366828 \\ \text { Email } & \text { cperez@gbt.tfo.upm.es }\end{array}$

Author Disclosure: The authors have no conflicts of interest

Running title: Neural Network Algorithm for On-Line Glucose Prediction 


\begin{abstract}
Background and Aims: Continuous glucose monitoring (CGM) devices could be useful for real-time management of diabetes therapy. In particular, CGM information could be used in real-time to predict future glucose levels in order to prevent hypo/lyyperglycemic events. This paper proposes a new on-line method for predicting future glucose concentration levels from CGM data.

Methods: The predictor is implemented with an artificial neural network model (NNM). The inputs of the NNM are the values provided by the CGM sensor during the preceding 20 minutes, while the output is the prediction of glucose concentration at the chosen prediction horizon $(\mathrm{PH})$ time. The method performance is assessed using datasets from two different CGM systems (9 subjects using the Medtronic Guardian and 6 subjects using the Abbott Navigator). Three different PH are used, i.e. 15, 30 and 45 minutes. The NNM accuracy has been estimated by using the root mean square error (RMSE) and prediction delay.

Results: The RMSE is around 10.18 and $27 \mathrm{mg} / \mathrm{dl}$ for 15,30 and 45 minutes of $\mathrm{PH}$, respectively. The prediction delay is around 4. 9 and 14 minutes for upward trends and 5, 15 and 26 minutes for downward trends, respectively. A comparison with a previously published technique, based on an autoregressive model (ARM) [1], has been performed. The comparison shows that, the proposed NNM is more accurate than the ARM, with no significant deterioration in the prediction delay.

Conclusions: The proposed NNM is a reliable solution for the on-line prediction of future glucose concentrations from CGM data
\end{abstract}




\section{Introduction}

One of the difficulties in achieving a good metabolic control is that patients have limited information about blood glucose for assessing the adequacy of the therapy for their daily actions. Insulin therapy has to respond dynamically to the glucose levels and the corresponding insulin demand. The factors that play an important role in the insulinghicose balance are: the diet. the plysical exercise, stress and illness, etc. For this reason, blood sugar levels may become harder to control by the patient himself [2].

Traditionally self-monitoring of blood glucose requires the drawing of a blood spot several times a day. The number of capillary blood glucose measurements (CBGM) is reduced mainly due to the pain associated with testing. Nowadays, the availability of continuous glucose monitoring (CGM), which provides information in real time, makes it possible to obtain a subcutaneous glucose reading every few minutes [3]. In this new scenario patients have to interpret a huge amount of continuous information in order to calculate the necessary insulin dose and bring blood sugar levels as close to normal as possible.

Most of the CGM systems available in the market are minimally invasive systems that measure the glucose in the interstitial fluid (ISF) compartment and not directly from plasma. Thus. there is a distortion between the glucose in the ISF with regard to the blood glucose levels, with a temporal delay that can become longer than 10 minutes [3]. A glucose predictor would enhance the CGM with valuable information on the expected glucose level in a time horizon. The predictor could aid the patient in anticipating his/her actions, but a predictor model is useful if the prediction horizon ( $\mathrm{PH})$ is long enough to allow the patients to anticipate their decisions, and that also depends on the relative delay between the CGM system measurement and the blood glucose value.

Artificial Neural Networks could be especially recommended to solve problems that cannot be easily described by mathematical algorithms that are highly non-linear or when the variables can evolve from the initial conditions. For these reasons neural network techniques have been used in the past for predicting glucose levels using CBGM values as input and also within glucose control systems. Some examples are reported below.

Sandham et al. [4] presented a neural network model (NNM) that used blood glucose capillary measurements, insulin doses and intake as input data, for predicting blood glucose levels. It also considered other relevant information. if available. like exercise. stress. illness or surgery. The network was evaluated with data from two 
patients.

Mougiakakou et al. [5] proposed a hybrid system based on the combination of a compartmental model (CM) and a NNM for blood glucose prediction. The CM generates an estimation of the insulin and intake effect and the NNM used this estimation and two previous CBGM to predict the subsequent blood glucose levels. The CM utilized information about insulin type and doses as well as the description of food intake. The network was evaluated with data from a Type 1 diabetes patient over a period of 69 days.

Volker Tresp et al. [6] also presented a hybrid CM-NNM system. The CM simulates the delay in carbolydrate intake absorption, the plysical exercise and the administered insulin dynamics. The NNM uses the CM estimation and the current and previous estimates of the blood glucose to predict the next glucose value. Data was used from one patient monitored over 63 days at 15-minute intervals.

All of the predictors referred to the use of self-CBGM [4-6] and required auxiliary details like insulin and intake data, hypoglycemic events and exercise among others. The availability of CGM opens a new scenario: glucose measurement is continuous information and any method suitable for time series analysis could be explored in the attempt to find a solution for the prediction problem [9].

Sparacino et al. compared the predictive performance of a first-order polynomial model [10] with that of a firstorder autoregressive model (ARM) [1], both with time-varying parameters. The inputs of the models were past glucose data. The models were evaluated with data of diabetic patients wearing the GlucoDay ${ }^{\circledR}$ CGM System (A. Menarini Diagnostics, Italy) that provides glucose levels every 3 minutes. The results demonstrated that glucose prediction was feasible with the two prediction methods and concluded that for the dataset used and a prediction interval of 30 minutes, the ARM was the more reliable for obtaining a clinically significant prediction performance.

Reifman et al. [11] used a 10th-order data-driven ARM. The inputs of the model were the previously observed glucose levels. It was trained and evaluated with data of the iSense ${ }^{\circledR}$ CGM system (iSense Corporation, Portland, Oregon). The CGM measurement rate was one sample per minute. The results demonstrated that the predictions for a PH of 30 minutes were quite accurate.

Palerm et al. [12] proposed an algorithm to predict glucose levels based on the estimation of glucose and its rate of change, using a Kalman filter. They applied the algorithm to predict hypoglycemia using data from a series of hypoglycemic clamps in which a CGMS ${ }^{\text {B }}$ System (Medtronic-Minimed, Northridge, CA) was employed [13]. For 
30 minutes of $\mathrm{PH}$ and an alarm threshold of $70 \mathrm{mg} / \mathrm{dl}$, the algorithm was effective in predicting hypoglycemia (sensitivity of $90 \%$ and specificity of $79 \%$ ).

Artificial Neural Network Models have also been applied in the prediction of glucose concentrations using CGM data. Pappada et al. [14] presented a NNM trained with data from the CGMS Medtronic System in combination with other data manually recorded by the patient in an electronic diary (i.e. CBGM, insulin dosages, carbohydrate intakes, hypoglycemic and hyperglycenic symptoms, lifestyle activities, events and emotional state). Unfortunately the prediction accuracy cannot be compared with that reported in other works in the literature $[1,10,11]$ because they calculated the mean percent absolute difference of the model's predictive abilities instead of the commonly used evaluation parameters. They concluded that the model tended to underestimate extreme hyperglycemic values and overestimate hypoglycemic values.

In this paper we propose a new real-time prediction algorithm based on a NNM for CGM. The model is trained using only CGM data as inputs. and the validation is performed by exploiting two datasets from two different commercial CGM devices. The results show that the accuracy of NNM prediction is satisfactory. Furthermore, the NNM is more accurate than the ARM developed by Sparacino et al. [1], with no significant deterioration in the prediction delay. The proposed NNM algorithm is therefore a reliable solution for the on-line prediction of future glicose concentrations based on CGM data.

\section{Methods}

\subsection{Subjects and Dataset}

The evaluation of the prediction algorithm was performed using data from the Guardian ${ }^{\circledR}$ Real-Time CGM System (Medtronic-Minimed, Northridge, CA) and the FreeStyle Navigator ${ }^{\sqrt{8}}$ CGM System (Abbott Laboratories. Abbott Park, Illinois). The Guardian ${ }^{\text {(3) }}$ provides a single output measurement every 5 minutes (288 samples per day). The FreeStyle Navigator ${ }^{\circledR}$ produces a single output measurement every minute (1440 samples per day).

The Guardian ${ }^{\circledR}$ dataset includes data from nine patients with Type 1 diabetes. The patients wore the CGM system intermittently for 72 hours/week over four weeks. The dataset includes 12 daily profiles for every patient: 6 full-day and 6 half-day recordings [15] 
The FreeStyle Navigator $^{(1)}$ dataset includes data from six patients with Type 1 diabetes. The patients wore the CGM system for around 72 hours. The dataset includes 2 complete daily profiles for every patient [16].

Figure I shows two profiles extracted from the respective datasets.

Remark 1. Before feeding the prediction algorithm, it was found convenient to reduce the effect of noise in the FreeStyle Navigator ${ }^{\text {(3) }}$ profiles ( 1 min. sampling) by pre-filtering them using a causal Kalman filtering method [17]. However. the quantitative assessment of the prediction methodology in Section 2.4 is done by taking the original profiles as reference.

\subsection{Description of the neural network model}

The network architecture has three layers with a first layer of 10 neurons and a second layer of 5 neurons [15] The transfer function is sigmoidal in both layers. Neurons are totally connected and feedforward. The output layer has one neuron with linear transfer function.

The network input information is the current glucose measurement and its timestamps together with a limited number of previous glucose samples from the CGM system. The NNM takes into account the glucose measurements up to 20 minutes before the current time. Since the sampling rate varies from one CGM system to another, the number of the NNM inputs is different for each dataset. The output of the network is the glucose prediction at the PH time.

Figure 2 shows the NNM architecture using the Guardian ${ }^{\circledR}$ data. In this case, the sensor registers the glucose every 5 minutes, so this model needs 5 CGM samples as inputs to work with a memory of 20 minutes. Using the FreeStyle Navigator ${ }^{(31}$, the NNM presents 20 CGM samples as inputs. The current time is provided as the order number of the sample throughout the day. 
Network parameters (weights and bias) are randomly initialized and, afterwards, are updated according to a backpropagation Levenberg-Marquardt optimization training algorithm. The training algorithm is applied in a batch mode. in which weights and biases are only updated when all the inputs and targets are presented. Three NNM have been trained to predict glucose after 15,30 and 45 minutes in order to evaluate the network performance depending on the $\mathrm{PH}$.

For each dataset, 3 subjects (each with 2 daily profiles) were used for training the NNM. Missing samples have been calculated using spline techniques to recover missing samples before using the glucose profiles for training. Patients in the training set did not take part in the validation.

\subsection{Description of the autoregressive model}

The autoregressive model proposed here is the same first order model used in [1], which corresponds to the timedomain equation

$$
\mathbf{u}_{\mathbf{1}}=\mathbf{a} \mathbf{u}_{\mathrm{i}-1}+\mathbf{w}_{\mathbf{1}}
$$

In eq.(1), $i=1,2, \ldots$ n denotes the order of glucose samples collected up to the $n$-th sampling time $t_{n}$ and $\left\{w_{i}\right\}$ is a random white noise process with zero mean and variance equal to $\sigma^{2}$. Letting $\theta=\left(a, \sigma^{2}\right)$ denote the vector of the parameters of the model. at each sampling time $t_{12}$ a new value of $\theta$ is determined by fitting past glucose data $u_{n}$. $\mathbf{u}_{12}$. $1, u_{n-2}, \ldots$ by a weighted linear recursive least squares algorithm. Once $\theta$ is determined. the model is used to calculate the prediction of glucose level $Q$ steps ahead, i.e. $\hat{\mathbf{u}}_{1+C}$. where $\mathrm{Q} \cdot \mathrm{T}_{\mathrm{s}}=\mathrm{PH}\left(\mathrm{T}_{\mathrm{s}}\right.$ is the sensor sampling period). All the past data participate. with different relative weights. in the determination of $\theta$. As in [1]. here we chose exponential weights, i.e. $\mu^{\mathrm{k}}$ is the weight of the sample taken $\mathrm{k}$ instants before the actual sampling time with $\mu$ termed forgetting factor. For more specific details on the algorithm, we refer the reader to [1].

A different optimal $\mu$ value has been chosen for each dataset and for each $\mathrm{PH}$, minimizing the root mean square error RMSE (mg/dl) between the original data of the training set and the predicted values obtained applying the ARM to it. The training set was the same as that for the NNM (see section 2.2 for details). 
For the Guardian ${ }^{\circledR}$ dataset this optimization step suggested the use of $\mu=0.695,0.795$, and 0.87 for $\mathrm{PH}=15,30$, and 45 minutes, respectively. For the FreeStyle Navigator ${ }^{\circledR}$ dataset, the parameters were $\mu=0.92,0.938$, and 0.985 for $\mathrm{PH}=15,30$, and 45 minutes.

\subsection{Evaluation Methodology}

Patients in the training set did not take part in the NNM and ARM validation. The validation set for the Guardian $^{3 i}$ monitor includes 6 patients and 6 profiles per patient. The validation set for The FreeStyle Navigator ${ }^{\text {B }}$ monitor includes 3 patients and 2 profiles per patient. The profiles in the training set have been suitably chosen in order to represent both low and high glucose levels, e.g. they contain several hypo and hyperglycemic events.

Figures 3 and 4 show the original CGM profile and the predictions from the NNM and ARM for 15, 30 and 45 minutes of PH for the same examples shown in Figure 1 (a) and (b).

To assess the performance of each model, we calculate the model accuracy and the model delay and we evaluate the influence of different $\mathrm{PH}(15,30$ and 45 minutes) on the two parameters.

The model accuracy has been evaluated as the RMSE (mg/dl) of the predicted profiles vs. the original ones.

The model delay has been estimated calculating the delays between the original and the predicted profiles when they cross three different thresholds, defined in the following way. First, peaks and nadirs are identified in the original sensor profile. Then, thresholds are placed at $25 \%, 50 \%$ and $75 \%$ of the nadir-to-peak $\left(P_{1}-P_{1}^{\prime}\right) /$ peak-to$\operatorname{nadir}\left(P_{2}-P_{2}^{\prime}\right)$ distance for positive / negative trends, respectively (figure 5). The final model delay is calculated as the average of threshold delays for every positive and negative trend. Notably, for the Navigator data, the calculated delay includes a contribution due to the use of the pre-filtering procedure described in Section 2.1 (Remark 1).

Remark 2. In order to identify threshold crossings more easily, given the retrospective nature of the assessment, the original and the predicted profiles have been filtered by a first order Butterworth low-pass filter. with normalized cut-off frequency $\omega_{11}=0.1$ and $\omega_{n}=0.03$ for Guardian ${ }^{\beta_{1}}$ and FreeStyle Navigator ${ }^{18}$ systems, respectively. 
The differences in RMSE between the Guardian ${ }^{\circledR}$ and the FreeStyle Navigator ${ }^{\circledR}$ systems are analyzed with the non-parametric Mann-Whitney U test.

\section{Results}

Tables 1 and 2 show the average prediction error. RMSE in mg/dl and the standard deviation (SD). for each model (NNM and ARM) when applied to the Guardian ${ }^{\text {Bi }}$ and the FreeStyle Navigator ${ }^{\text {Bi }}$ validation sets. The results are calculated for 15,30 and 45 minutes of $\mathrm{PH}$.

Tables 3 and 4 show the average delay in minutes and the SD. considering positive (upward) and negative (downward) trends.

The precision of the NNM and the ARM (RMSE values in Tables 1 and 2) are quite similar and very accurate for $\mathrm{PH}=15^{\circ}$ (around $10 \mathrm{mg} / \mathrm{dl}$ for both models). However considering $\mathrm{PH}=30$ and 45, the NNM is more accurate than the ARM presenting lower RMSE values ( $18 \mathrm{mg} / \mathrm{dl} \mathrm{vs.} 22 \mathrm{mg} / \mathrm{dl}$ for $\mathrm{PH}=30$ and $27 \mathrm{mg} / \mathrm{dl} \mathrm{vs.} 35 \mathrm{mg} / \mathrm{dl}$ for $\mathrm{PH}=45$ )

For $\mathrm{PH}=15$ the mean delay with the NNM is usually around 3 minutes longer than for the ARM. When the PH increases, for $\mathrm{PH}=30$ and 45 , the ARM is more rapid than the NNM in all cases except for upward trends and FreeStyle Navigator ${ }^{\text {B? }}$. The delay (tables 3 and 4) shows that the NNM is useful for $\mathrm{PH}=30$ and 45 in helping Type I diabetic patients to prevent hypo and lyperglycemic events.

It is interesting to note that both models are more rapid in upward than in downward slopes (tables 3 and 4 ). This could be explained by the fact that, for physiological reasons, the upward trends are usually more rapid than the downward trends, and the upward-to-downward changes are usually more marked than downward-to-upward changes. For this reason both models exhibit greater overshooting in the upward-to-downward zones and the subsequent downward trend is dogged by this effect, so both models exhibit a greater delay in crossing the threshold in the subsequent downward trend. 
The non-parametric Mann-Whitney $U$ test shows that the RMSE is lower for the Guardian ${ }^{\circledR}$ than for the FreeStyle Navigator ${ }^{\circledR}$ system both for the NNM and the ARM (NNM-15, NNM-30, NNM-45, ARM-15, ARM-30 and ARM-45). The differences were always significant $(p<0.01)$.

\section{Conclusions}

From the results. we can conclude that the NNM predictor is a reliable solution to the problem of predicting glucose values from CGM systems. The method presented here is totally causal and can be applied for on-line glucose prediction. The training algorithm can be executed on a common PC. For the training set size used (6 profiles), the training algorithm takes from 1.5 to 2.5 hours for Guardian ${ }^{18}$ data (it depends on the configuration of the learning algorithm parameters) and from 7.5 to 8.5 hours (because of the more frequent sampling rate) for FreeStyle Navigator ${ }^{\left(\frac{1}{6}\right.}$ data. It is important to point out that, once the NNM is trained, the algorithm that calculates the prediction could run in real time even on a portable PDA system.

The results obtained by applying the NNM are very encouraging. In this work. we successfully tested the possibility of applying the NNM by exploiting as input data only the previously collected CGM values. We have proved that, even if the NNM has been trained with a reduced number of profiles, coming from several subjects (excluding individual data) and representing a variety of situations (including hypo and hyperglycemic events), the prediction is sufficiently accurate. This fact allows us to speculate that if the training set is incremented. almost any changes in glucose profile due to individual metabolic fluctuations may be accurately assessed by the NNM.

However, the fact that only past CGM data is used as input of the NNM introduces some limitations, i.e. it does not allow the NNM to rapidly detect possible sudden changes in the time derivative of the signal due to intrinsic changes (i.e. individual metabolic fluctuations) as well as extrinsic changes (i.e. meal intake and/or insulin injections). Integrating into the model quantitative information concerning. for example, insulin injections and meal intakes could be beneficial to the prediction (i.e. it could also allow the use of a wider PH and facilitate tracking of rapid changes in glucose). However, how to do it is still rather unexplored in the literature (also because of the lack of these pieces of information in the majority of the available databases). 
At the present time, we are investigating new models that take into account intake and insulin delivery in different ways (as simple as possible for the patient) as input data. In order to improve the NNM structure, a new trial protocol has been designed in which meals and insulin delivery are tracked.

The results show that the prediction accuracy is better with Guardian ${ }^{18}$, even though its sample rate is lower than that of the FreeStyle Navigator ${ }^{\text {(ब) }}$.

Several other aspects could be studied in the future. For instance, since the quantitative performance of the predictor seems to be quite dependent on the specific CGM system, it might be interesting to evaluate the NNM with other CGM systems. It could be of interest to analyze the impact on predictors of different sampling rates and different noise variances. In addition, the possibility of predicting the blood glucose rather than the interstitial glucose could be taken into account, i.e. by combining the model with an on-line deconvolution procedure based on a physiological model for comparing plasma and interstitial fluid glucose kinetics.

\section{Acknowledgements}

Prof. Boris Kovatchev (University of Virginia Health System. Charlottesville. VA) is acknowledged for having provided part of the data published in [16].

\section{References}

[1] G. Sparacino, F. Zanderigo, S. Corazza, A. Maran, A. Facchinetti and C. Cobelli, "Glucose Concentration can be Predicted Ahead in Time From Continuous Glucose Monitoring Sensor Time-Series," Biomedical Engineering, IEEE Transactions on, 2007, vol. 54, pp. 931-937.

[2] The American Association of Clinical Endocrinologists, AACE., "Medical guidelines for the management of diabetes mellitus: the AACE system of intensive diabetes self-management," Endocrine Practice, 2007, vol. 13.

[3] S. K. Garg, H. K. Hoff and H. P. Chase, "The role of contimous glucose sensors in diabetes care," Endocrinology \& Metabolism Clinics of Nonth America, 2004, vol. 33, pp. 163-173. 
[4] W. A. Sandham, D. Nikoletou, D. J. Hamilton, K. Patterson, A. Japp and C. MacGregor, "Blood glucose prediction for diabetes therapy using a recurrent artificial neural network," in L European Signal Processing Conference (EUSIPCO), Rhodes, 1998 , pp. $673-676$.

[5] S. G. Mougiakakou, K. Prountzou and K. S. Nikita, "A real time simulation model of glucose-insulin metabolism for type 1 diabetes patients," in 27th Annual Intermational Conference of the Engineering in Medicine and Biology Society (IEEEEMBS), 2005, pp. 298-301.

[6] V. Tresp, T. Briegel and J. Moody, "Neural-network models for the blood glucose metabolism of a diabetic," Neural Networks, IEEE Transactions on, 1999, vol. 10, pp. 1204-1213.

[7] 2. Trajanoski and P. Wach, "Neural predictive controller for insulin delivery using the subcutaneous ronte," Biomedical Engineering, IEEE Transactions on, 1998, vol. 45, pp. 1122-1134.

[8] S. G. Mongiakakou and K. S. Nikita, "A neural network approach for insulin regime and dose adjustment in type 1 diabetes," Diabetes Techmologv and Therapeutics, 2000, vol. 2, pp. 381-389.

[9] T. Bremer and D. A. Gough, "Is blood glucose predictable from previous values? A solicitation for data," Diabetes, 1999. vol. $48, \mathrm{pp}, 445-451$.

[10] G. Sparacino, F. Zanderigo, A. Maran and C. Cobelli, "Continuous glucose monitoring and hypo/hyperglycaemia prediction," Diabetes Reseanch and Clinical Practice, 2006, vol. 74, pp. \$160-\$163.

[11] I. Reifman, S. Rajaraman, A. Gribok and W. K. Ward, "Predictive monitoring for improved management of glucose levels," Joumal of Diabetes Science and Technology, 2007, vol, 1, pp. 478-486.

[12] C. C. Palerm, J. P. Willis, J. Desemone and B. W. Bequette, "Hypoglycemia Prediction and Detection Using Optimal Estimation," Diabetes Technology \& Therapeutics, 2005, vol. 7, 1, pp. 3-14.

[13] C. C. Palerm and B. W. Bequette, "Hypoglycemia detection and prediction using continuous glucose monitoring - a study on hypoglycemic clamp data," Joumal of Diabetes Science and Technologv, 2007, vol. 1, pp. 624-629.

[14] S. M. Pappada, B. D. Cameron and P. M. Rosman, "Development of a Neural Network for Prediction of Glucose Concentration in Type I Diabetes Patients," J Diabetes Sci Technol, 2008, vol. 2, pp. 792-801.

[15] C. Pérez-Gandía, M. E. Hernando, C. O. S. Sorzano, A. Otero-Quintana, G. Garcia-Sáez, A. Rodriguez-Herrero, M. Rigla, A. de Leiva and E. J. Gómez, "Neural networks for glucose prediction in continuous glucose monitoring," in 1 st Conference on Advanced Techologies \& Treatments for Diabetes (ATTD), Prague, 2008, pp. 112.

[16] B. P. Kovatchev, L. A. Gonder-Frederick, D. J. Cox and W. L. Clarke, "Evaluating the Accuracy of Continuous GlucoseMonitoring Sensors: Continuous glucose-error grid analysis illustrated by TheraSense Freestyle Navigator data," Diabetes Care, 2004, vol. 27, pp. 1922-1928. 
[17] A. Facchinetti, G. Sparacino and C. Cobelli, "Real time adaptive Kalman filtering for continuous glucose monitoring noise removal," in 8th Diabetes Technology Meeting (DTM), Bethesda, 2008, pp. A58. 

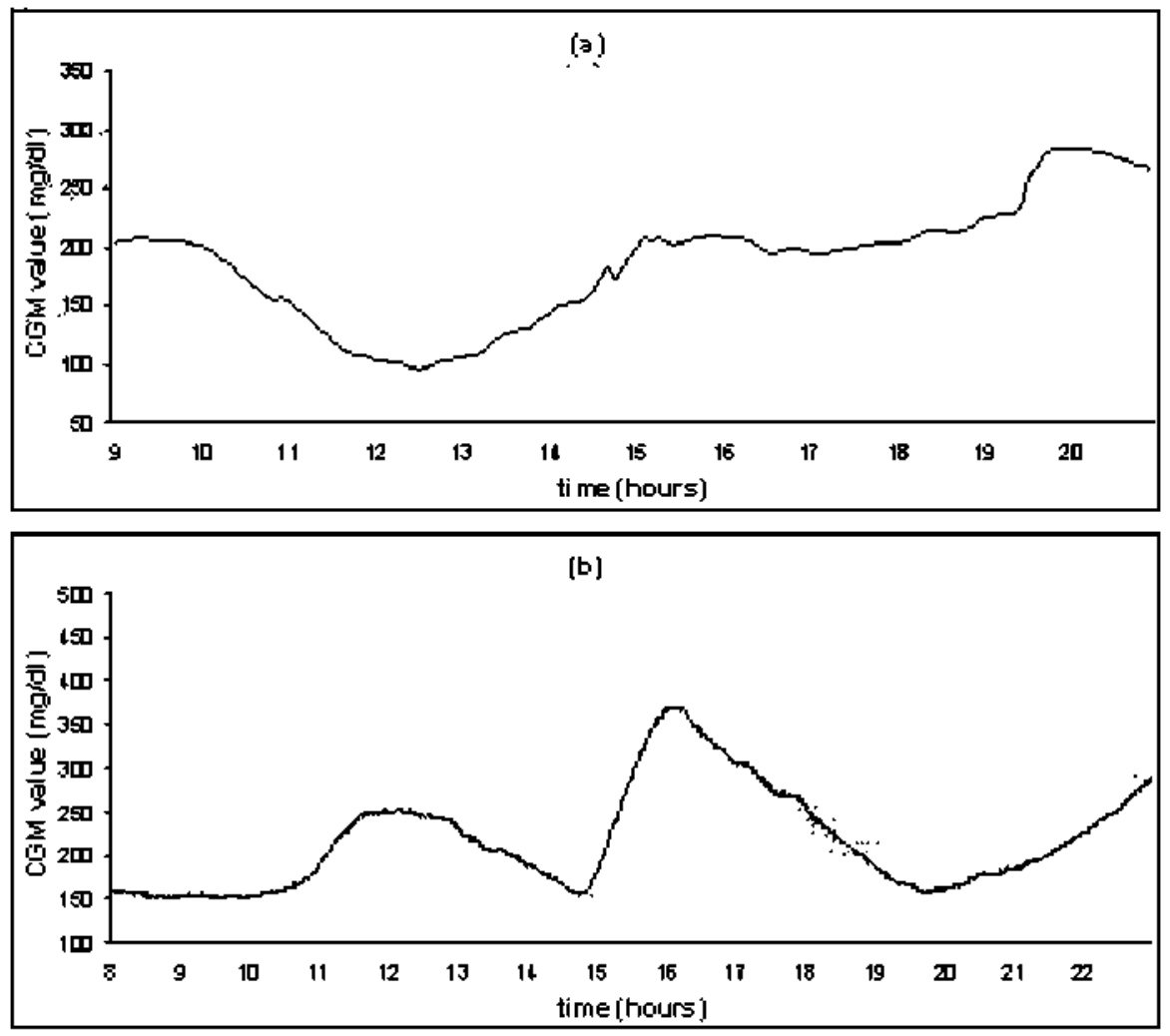

Figure 1. Two representative CGM profiles from Guardian@ (a) and from FreeStyle Navigator@ (b) $118 \times 105 \mathrm{~mm}(96 \times 96 \mathrm{DPI})$ 


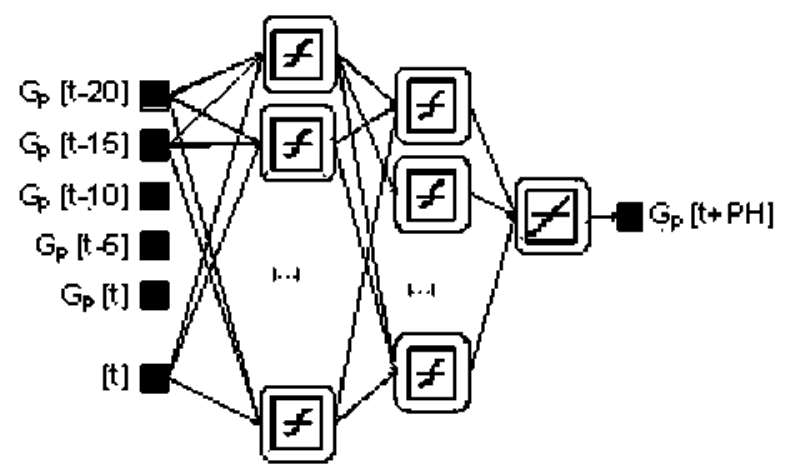

Figure 2. NNM architecture when the Guardian® sensor (5 min sampling) is used $81 \times 51 \mathrm{~mm}(96 \times 96 \mathrm{DPI})$ 

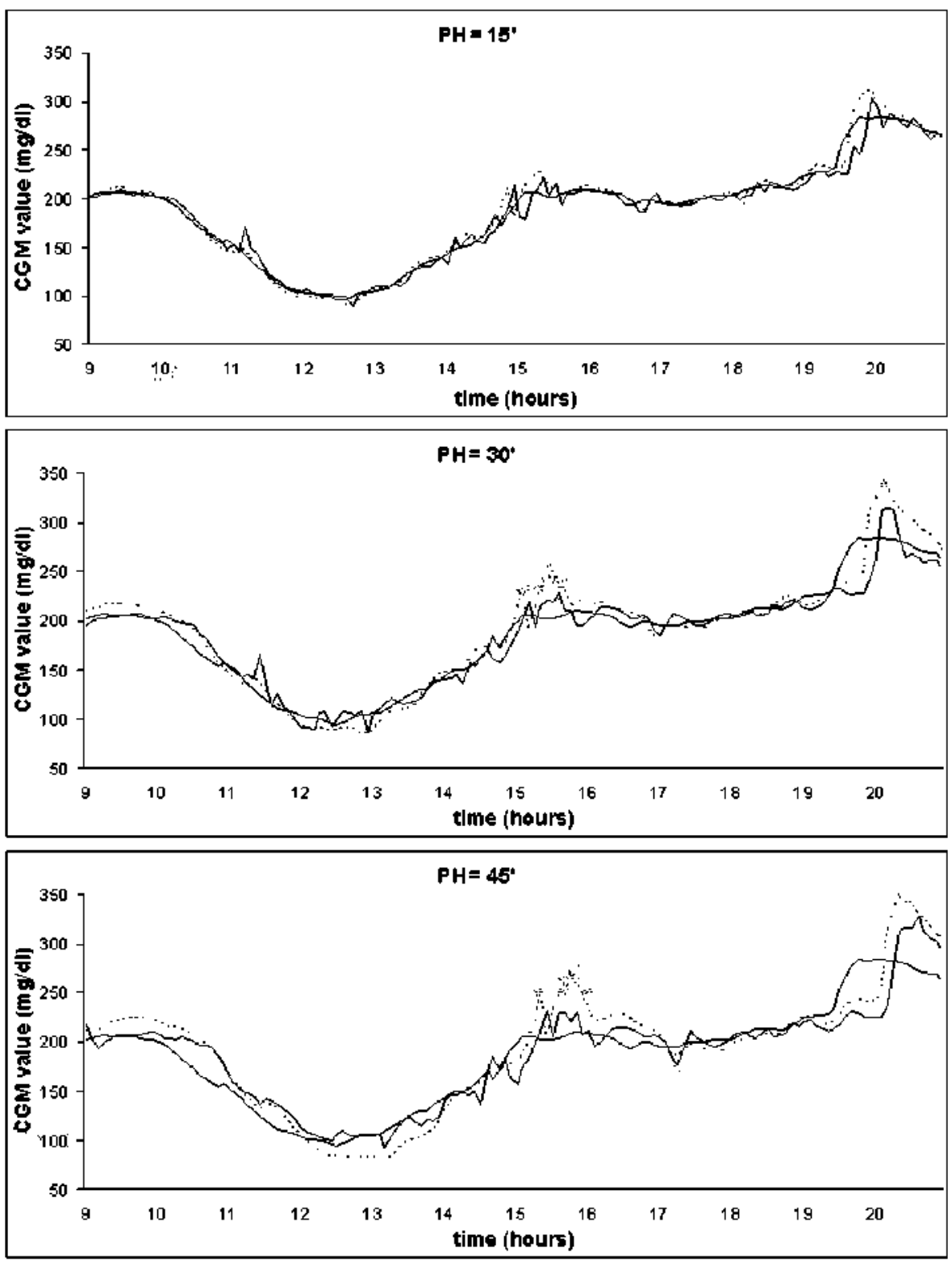

Figure 3. Original Guardian( profile (-), NNM (-) and ARM (- - ) predictions $164 \times 218 \mathrm{~mm}(99 \times 99 \mathrm{DPI})$ 

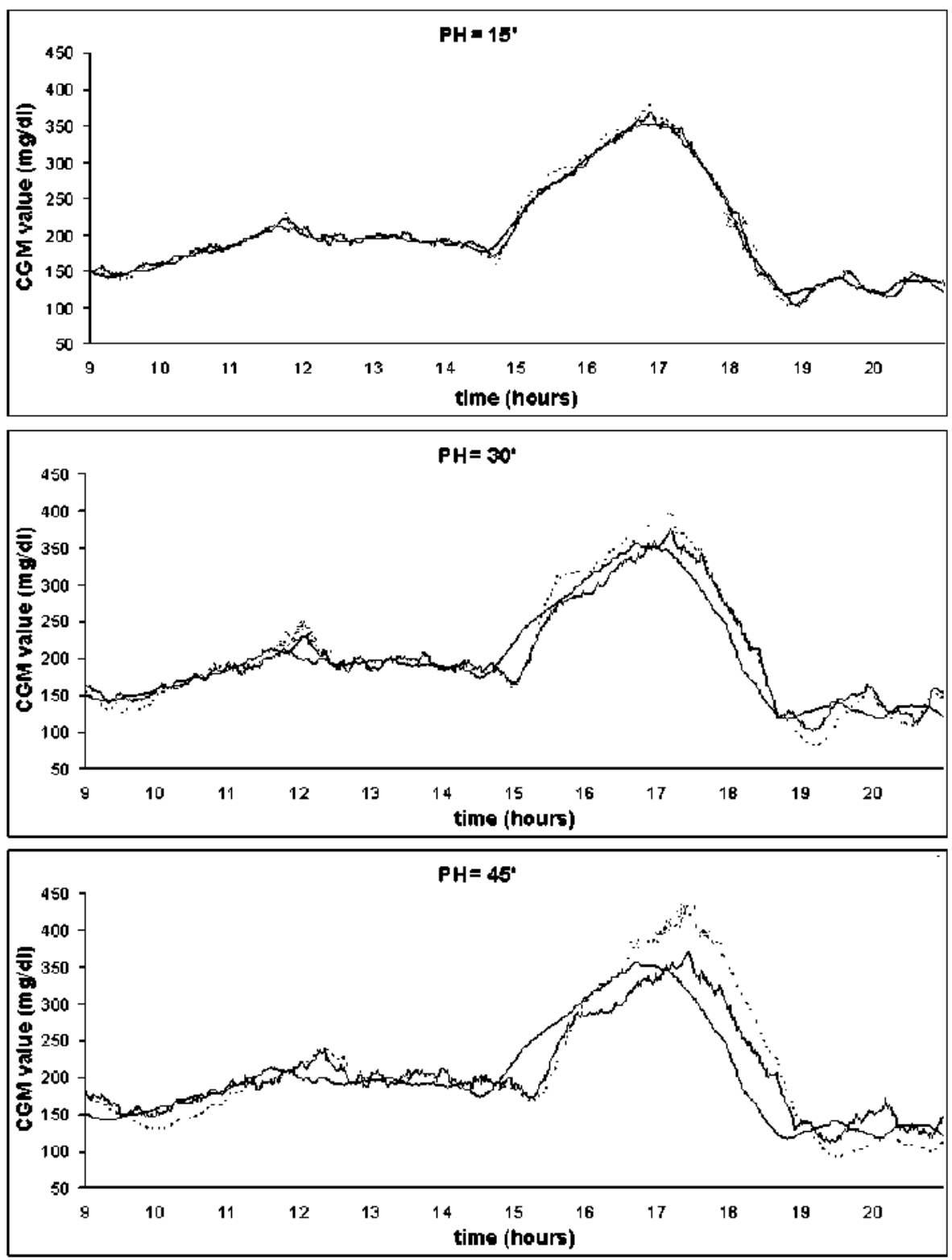

Figure 4. Original FreeStyle Navigator $($ profile (-), NNM (-) and ARM (- - ) predictions $164 \times 218 \mathrm{~mm}(99 \times 99 \mathrm{DPI})$ 


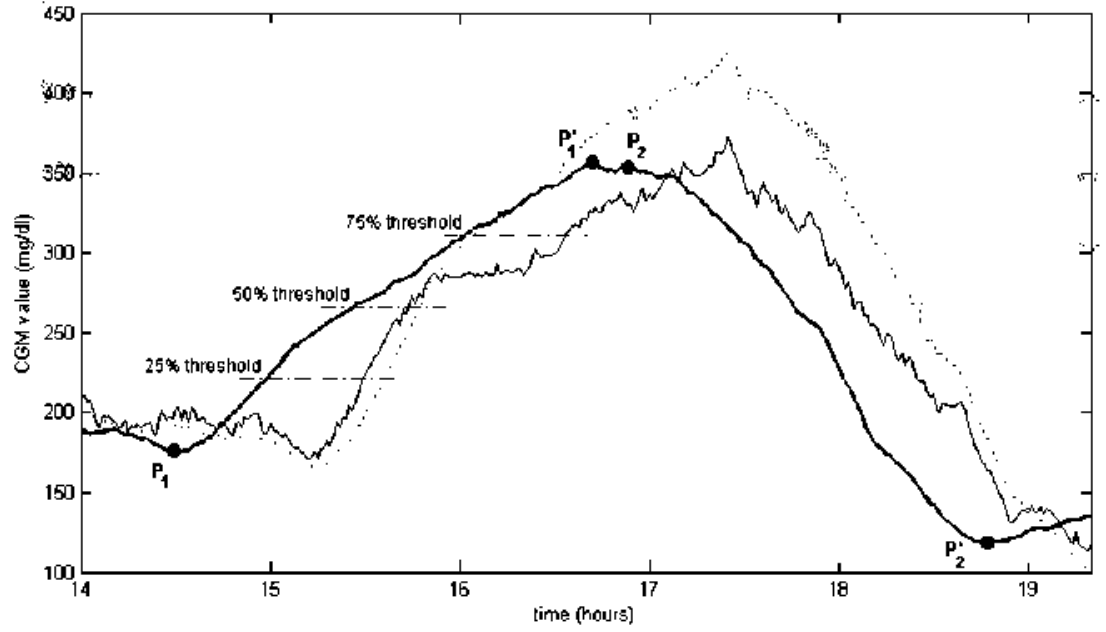

Figure 5. Algorithm to calculate the model delay $234 \times 123 \mathrm{~mm}$ ( $96 \times 96 \mathrm{DPI})$ 


\begin{tabular}{|c|c|c|c|}
\hline & $\mathbf{1 5}$ & $\mathbf{3 0}$ & $\mathbf{4 5}$ \\
\hline NNM & $9.74 \pm 2.71$ & $17.45 \pm 5.44$ & $25.08 \pm 8.73$ \\
\hline ARM & $9.26 \pm 2.97$ & $20.27 \pm 7.27$ & $30.30 \pm 11.89$ \\
\hline
\end{tabular}

Table 1. RMSE \pm SD $(\mathrm{mg} / \mathrm{dl})$ for Guardian( $104 \times 26 \mathrm{~mm}(96 \times 96 \mathrm{DPI})$ 


\begin{tabular}{|c|c|c|c|}
\hline & $\mathbf{1 5}$ & $\mathbf{3 0}$ & $\mathbf{4 5}$ \\
\hline NNM & $10.38 \pm 3.15$ & $19.51 \pm 5.53$ & $29.07 \pm 6.77$ \\
\hline ARM & $10.46 \pm 3.55$ & $24.36 \pm 8.97$ & $39.36 \pm 10.38$ \\
\hline
\end{tabular}

Table 2. RMSE \pm SD (mg/dl) for FreeStyle Navigator( $104 \times 26 \mathrm{~mm}(96 \times 96$ DPI $)$ 


\begin{tabular}{|l|c|c|c|c|}
\hline \multicolumn{2}{|c}{15} & $\mathbf{3 0}$ & $\mathbf{4 5}$ \\
\hline \multirow{2}{*}{ Upward } & NNM & $3.92 \pm 1.21$ & $11.65 \pm 4.17$ & $16.46 \pm 4.79$ \\
\cline { 2 - 5 } & ARM & $0.75 \pm 0.28$ & $6.26 \pm 1.35$ & $12.59 \pm 3.01$ \\
\hline \multirow{2}{*}{ Downward } & NNM & $5.10 \pm 1.65$ & $15.92 \pm 3.64$ & $23.82 \pm 5.13$ \\
\cline { 2 - 5 } & ARM & $1.30 \pm 0.38$ & $8.87 \pm 0.44$ & $19.15 \pm 1.41$ \\
\hline
\end{tabular}

Table 3. Delay \pm SD (minutes) for Guardian(B) $128 \times 41 \mathrm{~mm}(96 \times 96 \mathrm{DPI})$ 


\begin{tabular}{|c|c|c|c|c|}
\hline & & 15 & 30 & 45 \\
\hline \multirow{2}{*}{ Upward } & NNM & $4.58 \pm 1.42$ & $7.26 \pm 3.34$ & $11.50 \pm 6.85$ \\
\hline & ARM & $1.74 \pm 0.50$ & $5.04 \pm 1.18$ & $19.55 \pm 7.48$ \\
\hline \multirow{2}{*}{ Downward } & NMM & $5.37 \pm 2.16$ & $14.56 \pm 3.81$ & $28.67 \pm 4.62$ \\
\hline & ARM & $3.18 \pm 1.58$ & $9.51 \pm 3.56$ & $18.30 \pm 7.22$ \\
\hline
\end{tabular}

Table 4. Delay \pm SD (minutes) for FreeStyle Navigator $(R)$ $129 \times 39 \mathrm{~mm}(96 \times 96 \mathrm{DPI})$ 